\title{
Structural analysis of poly(ethylene terephthalate) during uniaxial drawing above the glass transition temperature
}

\author{
Kazuyuki Okada ${ }^{1}$, Takuji Higashioji ${ }^{2}$, Takeshi Nakagawa ${ }^{1}$, Hirohito Uchida ${ }^{2}$, Kenta Takahashi ${ }^{2}$, Rintaro Inoue ${ }^{3}$, \\ Koji Nishida ${ }^{3}$ and Toshiji Kanaya ${ }^{3}$
}

We performed small-angle and wide-angle X-ray scattering (SAXS and WAXS) measurements on poly(ethylene terephthalate) (PET) uniaxially drawn from an amorphous state above the glass transition temperature. The measurements were performed at a new beamline (BL), BL03XU, which is dedicated to the development of advanced soft materials and belongs to the Advanced Softmaterial Beamline Consortium. The BL03XU BL has a very high intensity, wide-dynamic range and low background. The characteristics of the BL03XU BL enable us to conduct a very precise and detailed analysis of the data. In this report, we studied the change in the lamellar structure during uniaxial drawing using detailed analysis of two-dimensional (2D) SAXS patterns and found that the X-shaped pattern appeared before the so-called 4-spot pattern of PET in the 2D SAXS data. The $X$-shaped pattern and the 4-spot pattern were considered to be due to isolated single lamellae and stacked lamellae, respectively. We performed a calculation to reproduce the observed 2D SAXS patterns, focusing especially on the 4-spot patterns, using a simple lamellar model that took into account the lamellar size, the lamellar spacing and the orientation distribution of the lamellar stacking direction. Based on the results of the calculation, we discussed the development of the lamellar structure during the drawing process.

Polymer Journal (2013) 45, 50-56; doi:10.1038/pj.2012.197; published online 21 November 2012

Keywords: lamellar crystal; poly(ethylene terephthalate); SAXS; uniaxial drawing; WAXS

\section{INTRODUCTION}

Biaxially stretched poly(ethylene terephthalate) (PET) films are currently the most widely used polymeric substrate material in the fields of display technologies, electronic devices, storage media, electronic insulators, automobiles, molding, packaging, printing and architecture, because the films show high strength, durability, transparency, flexibility and good surface properties. In industry, biaxially stretched PET films are normally prepared by the sequential drawing of amorphous PET in two directions, instead of by the simultaneous drawing of the PET in both directions. The sequential biaxial drawing leads to orientation and crystallization of the PET molecules in an amorphous region, resulting in tensilized films. After the biaxial drawing, a heatsetting treatment is performed to generate heat-induced crystallization. The temperature or drawing rate during the drawing has to be controlled so that the films are uniformly drawn without tearing. Therefore, it is very important to study the relationship between the structural formation and the conditions during drawing, such as the temperature and drawing rate. One of the most important structures that affect the film tearing is the crystalline lamellar structure and/or the higher order structure consisting of lamellae.
Because of the importance of PET films in industry, a number of studies on the deformation of PET during the drawing process have been conducted. ${ }^{1-14}$ Asano et al. ${ }^{1}$ studied the structural changes during isothermal annealing of cold-drawn PET using wide-angle X-ray scattering (WAXS) and small-angle X-ray scattering (SAXS). The results are very revealing. In the WAXS measurements, they observed that the initially formed nematic phase (or oriented amorphous phase) transformed into the smectic phase and finally into the triclinic crystalline phase during annealing. In the WAXS patterns of the triclinic phase, some reflections are located at higher and lower $q$ values than the normal layer line positions, indicating that the crystals are tilted in the same direction with respect to the unit cell. This type of orientation is known as a 'tilted orientation' and was first studied by Daubeny et al. ${ }^{15}$ In the SAXS measurements, they observed the so-called 4-spot pattern, showing the inclination of the surface of the lamellar crystals. The inclination of the surface layer was explained in terms of the molecular tilt mechanism. ${ }^{1}$ It is noted that the 2-spot pattern in SAXS, which is similar to the so-called long period peak in many polymers, is formed in PET during hot drawing at $110{ }^{\circ} \mathrm{C},{ }^{6}$ suggesting that the PET chains are highly inclined while

${ }^{1}$ Materials Science Laboratories, Toray Research Center, Inc., Shiga, Japan; ${ }^{2}$ Films and Film Products Research Laboratories, Toray Industries, Shiga, Japan and ${ }^{3}$ Institute for Chemical Research, Kyoto University, Kyoto, Japan

Correspondence: K Okada, Materials Science Laboratories, Toray Research Center, Inc., 3-3-7, Sonoyama, Otsu, Shiga 520-8567, Japan.

E-mail: kazuyuki_okada@trc.toray.co.jp

Received 3 September 2012; revised 25 September 2012; accepted 25 September 2012; published online 21 November 2012 
the lamella normal becomes parallel to the drawing direction, but the mechanism for this is not fully understood at the moment. Recently, Kawakami et al. ${ }^{2-6}$ studied a superstructure consisting of lamellar crystals and microfibrils of PET during uniaxial drawing at 90 and $100^{\circ} \mathrm{C}$, which is above the glass transition temperature, using in-situ SAXS and WAXS. They found an X-shaped scattering in the SAXS patterns at the beginning of the drawing, followed by the 4-spot pattern that was observed in Asano's experiments, ${ }^{1}$ showing that the 4-spot pattern is formed during uniaxial drawing above the glass transition temperature. Note that Shimizu et al. ${ }^{7}$ also found the $\mathrm{X}$-shaped scattering before Kawakami's experiments. Their results are very helpful in understanding the structural development process of PET during uniaxial drawing. However, detailed information on the lamellar structure, the orientation and the distribution of orientation is lacking. Therefore, in this study, we focused our attention on the lamellar structure, the orientation and the distribution of orientation during uniaxial drawing of PET above the glass transition temperature. For this purpose, we performed ex-situ SAXS and WAXS measurements using a high-intensity synchrotron radiation X-ray beamline (BL) (BL03XU) at SPring- $8^{16}$ in combination with a high sensitivity and large dynamic range detector. The very low background at the BL also enabled us to carry out detailed analysis of the SAXS patterns. For detailed and quantitative analyses, we performed a simple model calculation to reproduce the observed two-dimensional (2D) SAXS patterns, taking into consideration the lamellar size, lamellar period, orientation of the lamellar stacking angle and their distributions. Based on the results, we will discuss the structural development of PET during uniaxial drawing above the glass transition temperature.

\section{EXPERIMENTAL PROCEDURES}

\section{Samples}

PET samples were prepared by Toray Industries in Japan. The weight-averaged molecular weight $\left(M_{\mathrm{W}}\right)$ of the samples was $30000 \mathrm{~g} \mathrm{~mol}^{-1}$, and the molecular weight distribution was $\sim 2$ in terms of $M_{\mathrm{w}} / M_{\mathrm{n}}$, where $M_{\mathrm{n}}$ was the numberaveraged molecular weight. Molten PET was extruded at temperatures between 280 and $300{ }^{\circ} \mathrm{C}$, and the undrawn sheet was obtained after cooling to ambient temperature on a casting drum. Before drawing, the sample was completely amorphous, with no detectable crystallinity by WAXS and differential scanning calorimetry. The sample was uniaxially drawn using a stretching machine at 90 and $100^{\circ} \mathrm{C}$, which is above the glass transition temperature of $80^{\circ} \mathrm{C}$, which was determined by differential scanning calorimetry at a heating rate of $10{ }^{\circ} \mathrm{Cmin}^{-1}$. After a given draw ratio was reached at a drawing rate of $10 \%$ per min, the sample was quenched to room temperature in the machine. The film was $\sim 0.3 \mathrm{~mm}$ thick.

\section{SAXS and WAXS measurements}

SAXS and WAXS measurements were carried out at the new BL BL03XU (frontier softmaterial $\mathrm{BL})^{16}$ at the synchrotron radiation X-ray facility SPring-8, which is dedicated to the development of advanced soft materials and belongs to the Advanced Softmaterial BL Consortium. The wavelength $\lambda$ was $0.1 \mathrm{~nm}$. The sample films were mounted in the sample holder in the normal transmission geometry, that is, the film surface normal was parallel to the X-ray beam. An R-AXIS VII imaging plate (Rigaku Co., Tokyo, Japan) was used as a detector system for the SAXS measurements, which has a high sensitivity and a large dynamic range $\left(\sim 10^{6}\right)$. The acquisition time for the SAXS measurements was 10 s. For the WAXS measurements, a Flat Panel (Hamamatsu Photonics K. K., Hamamatsu, Japan) was used, and the acquisition time was $20 \mathrm{~ms}$. The sampleto-detector distances were 2204 and $67.4 \mathrm{~mm}$ for the SAXS and WAXS, respectively. The $q$ ranges covered in the SAXS and WAXS measurements were $0.04-3.5 \mathrm{~nm}^{-1}$ and $6-30 \mathrm{~nm}^{-1}$, respectively, where $q$ is the length of a scattering vector defined by $q=4 \pi \sin (\theta) / \lambda$ ( $2 \theta$ being the scattering angle). The scattering angle was calibrated using a $\mathrm{CeO}_{2}$ standard from the National Institute of
Standards and Technology for WAXS and a silver behenate (AgBe) standard and a chicken collagen standard for SAXS. The SAXS data were corrected for the absorption of the sample and the background scattering from the air and the windows of vacuum passes, and the data were normalized to account for the sample thickness. In the figures of the 2D SAXS and WAXS patterns, we provided scale bars in $q$ to show the rough ranges of $q$. Speaking accurately, they are not precise in linearity but are very convenient for the reader. Note that the data reductions were performed using precise $q$ values.

\section{RESULTS AND DISCUSSION}

\section{SAXS and WAXS patterns}

The observed 2D SAXS and WAXS patterns of the PET films at several draw ratios at 90 and $100{ }^{\circ} \mathrm{C}$ are shown in Figures la and d. At the beginning of the drawing, up to the ratio of $200 \%$, both the SAXS and WAXS patterns show an isotropic amorphous phase. In the SAXS pattern at $250 \%$, a weak X-shaped pattern is observed at $100^{\circ} \mathrm{C}$ while a 4-spot pattern is observed at $90^{\circ} \mathrm{C}$ in addition to the $\mathrm{X}$-shaped pattern, suggesting that the crystallization rate at $90^{\circ} \mathrm{C}$ is higher than that at $100{ }^{\circ} \mathrm{C}$. Note that it is hard to see the weak scattering intensity in the $2 \mathrm{D}$ patterns, so we confirmed the anisotropy of the weak scattering in plots of the $1 \mathrm{D}$ scattering intensity as a function of the azimuthal angle. In quiescent crystallization, generally speaking, the crystallization rate increases with temperature in the so-called cold crystallization region, ${ }^{17}$ but the data from the uniaxial drawing process contradict this prediction. This contradiction might be because it is harder for aligned polymer chains to relax during drawing at a lower temperature due to their low mobility, resulting in orientation-induced crystallization. In the WAXS patterns at $250 \%$, some clear Bragg diffractions from the (010), (-110) and (100) planes in the triclinic form are observed at $90^{\circ} \mathrm{C}$, whereas no Bragg peaks, but an oriented broad amorphous peak, are observed at $100^{\circ} \mathrm{C}$, confirming the slower crystallization at $100{ }^{\circ} \mathrm{C}$.

At draw ratios above $250 \%$, the 4 -spot patterns appear at the high $q$ end of the streak in the X-shaped patterns in the SAXS data at both 90 and $100^{\circ} \mathrm{C}$, which is assigned to the stacking of inclined lamellar crystals, ${ }^{1}$ although the shapes of the 4 -spot patterns are quantitatively different from each other. This difference will be discussed later. The Bragg diffractions from the $(010),(-110)$ and $(100)$ planes in the triclinic form above $250 \%$ are displaced from the equator line, showing the tilted orientation of the polymer chains as reported by Asano et al. ${ }^{1}$ The displacement of the Bragg peaks decreases with the draw ratio, meaning that the chain titling increases with the draw ratio, especially at $90^{\circ} \mathrm{C}$.

In the present measurements at BL03XU, we observed 2D SAXS patterns with a very low background, even in the very low $q$ region near the beam stop. This enabled us to identify the very weak $\mathrm{X}$-shaped streak patterns at high draw ratios above $300 \%$, which is demonstrated in Figure 2 for $250 \%$ at $90{ }^{\circ} \mathrm{C}$ and $350 \%$ at $100^{\circ} \mathrm{C}$. Kawakami et al. ${ }^{5}$ also reported that the X-shaped pattern was observed in the initial crystallization process in orientated PET. What is the molecular origin of this X-shaped pattern? As shown above, the $\mathrm{X}$-shaped pattern appeared at the beginning of crystallization and the 4-spot pattern overlapped the X-shaped pattern at the high $q$ end of the streaks, suggesting stacking of the inclined lamellae. If the 4-spot pattern is caused by stacking of the inclined lamellae, it is possible that the X-shaped pattern originated from an isolated lamella. Using this idea, we analyzed the X-shaped pattern to estimate the size of an isolated single lamella. The intensity profile perpendicular to the streak in the X-shaped pattern has a valley at a certain $q_{\mathrm{xv}}$. If we assume that the valley is caused by the form factor of an isolated lamella, its length $L_{\mathrm{x}}$ can be roughly 

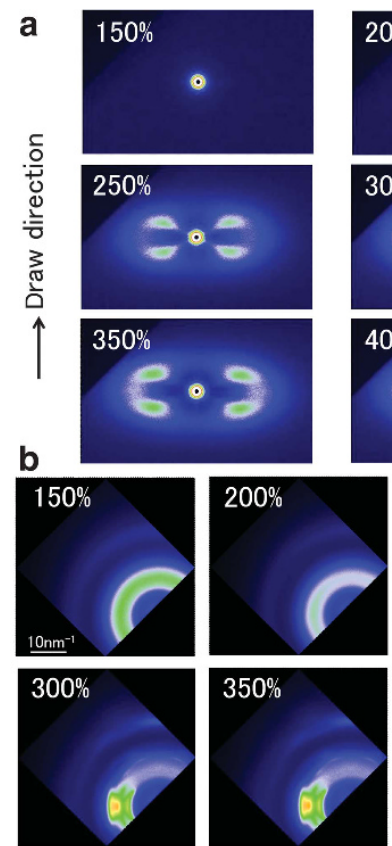
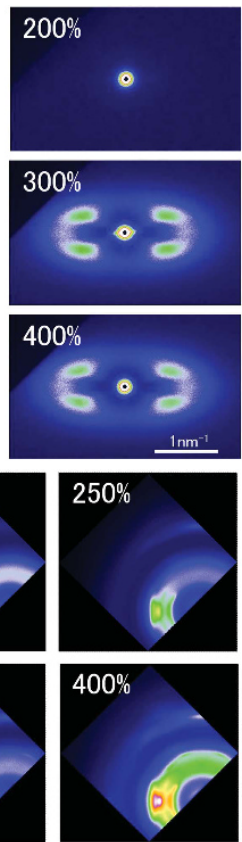
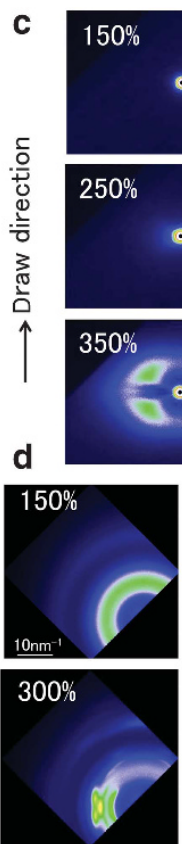
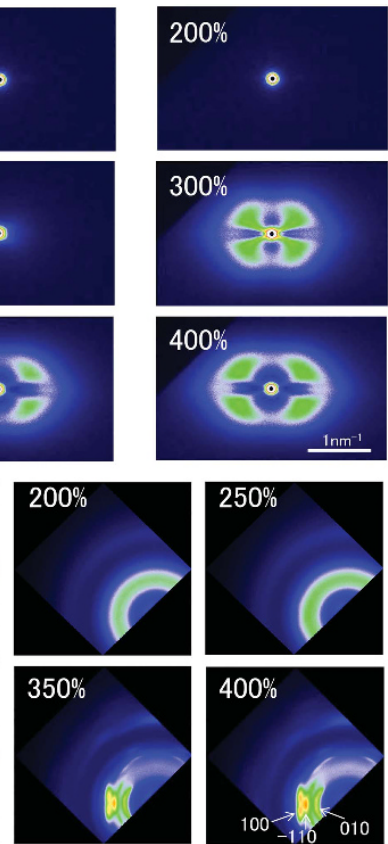
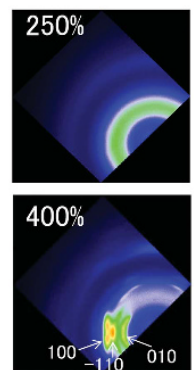

Figure 1 Two-dimensional (2D) small-angle X-ray scattering (SAXS) and wide-angle X-ray scattering (WAXS) patterns taken during uniaxial drawing at 90 and $100{ }^{\circ} \mathrm{C}$ at various draw ratios. (a) SAXS at $90^{\circ} \mathrm{C}$, (b) WAXS at $90^{\circ} \mathrm{C}$, (c) SAXS at $100^{\circ} \mathrm{C}$ and (d) WAXS at $100^{\circ} \mathrm{C}$.
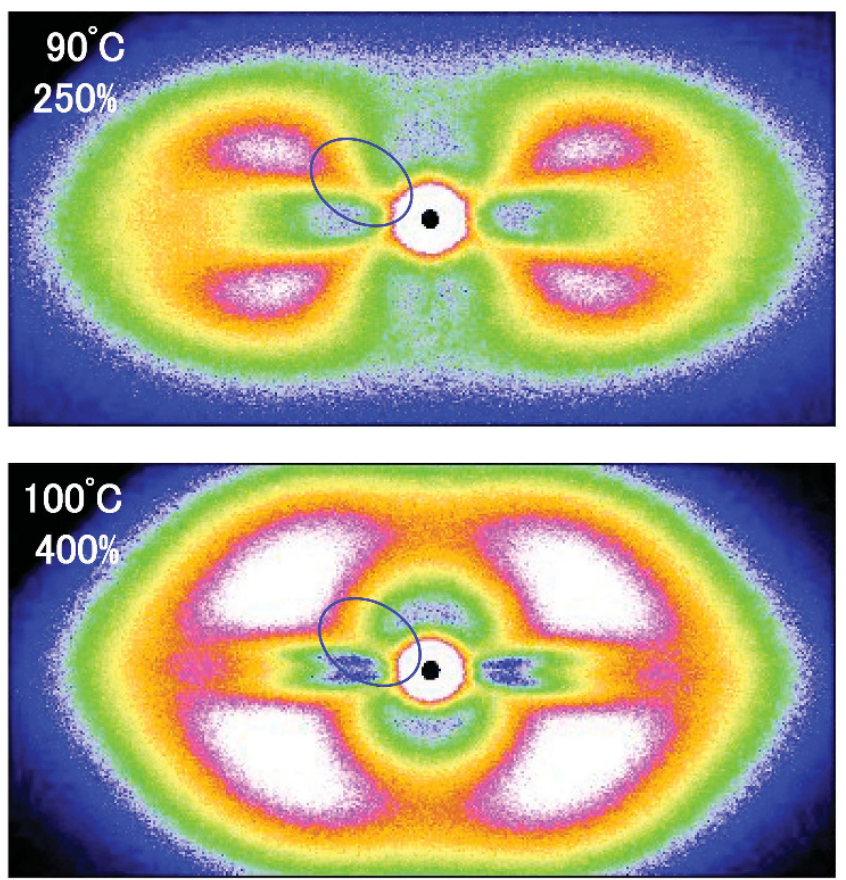

$1 \mathrm{~nm}^{-1}$

Figure 2 X-shaped patterns in the small-angle X-ray scattering (SAXS) images, which are indicated by the blue ovals. The color scale has been changed from Figure 1 to emphasize the X-shaped pattern. estimated as $L_{\mathrm{x}}=2 \pi / q_{\mathrm{xv}}$, although the value depends on the models used in the analysis. The length evaluated from $L_{\mathrm{x}}=2 \pi / q_{\mathrm{xv}}$ was $\sim 80 \mathrm{~nm}$ for the draw ratio of $400 \%$ at $100^{\circ} \mathrm{C}$. The lamellar thickness was also estimated using the intensity profile parallel to the streak of the X-shaped pattern and the Guinier method ${ }^{18}$ assuming that the cross-section of the isolated lamella was a sphere and the diameter of the sphere was the thickness of the lamella. The estimated value of the thickness is $\sim 6 \mathrm{~nm}$ at the drawing ratio of $400 \%$ at $100^{\circ} \mathrm{C}$. These length and the thickness values of the isolated lamella will be discussed later.

\section{Invariant and long period}

For further discussion, the apparent invariants (the integrated intensities) of the SAXS patterns, the long period and the azimuthal angle dependence of the 4-spot pattern intensity are evaluated. The apparent invariant for the X-shaped pattern was calculated as follows. First, we calculated the intensity profile along the streak in the $\mathrm{X}$-shaped pattern. In the low $q$ range, the intensity profile was smoothly extrapolated to $q=0$, using a Gauss function to merge it with the Guinier regime and neglecting any large-scale structure near the beam stop. In the high $q$ range, we tried to separate out the intensity of the 4-spot pattern, which was very high above the draw ratio of $300 \%$, and it was impossible. Hence, we limited the integration range up to $q=0.44 \mathrm{~nm}^{-1}$ and the azimuthal angle range from 10 to $45^{\circ}$.

Figures $3 \mathrm{a}$ and $\mathrm{b}$ show the apparent invariant of the 4-spot and the $\mathrm{X}$-shaped patterns, which correspond to the stacked lamellae and the single lamella, as a function of the draw ratio. The invariant of the 4-spot pattern first increases with the draw ratio at 90 and $100^{\circ} \mathrm{C}$, and 
a

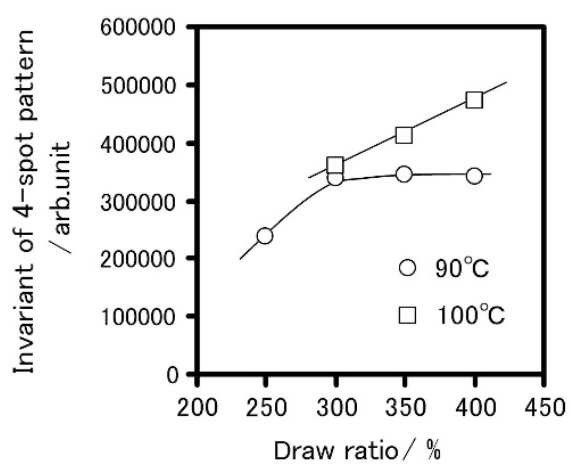

b

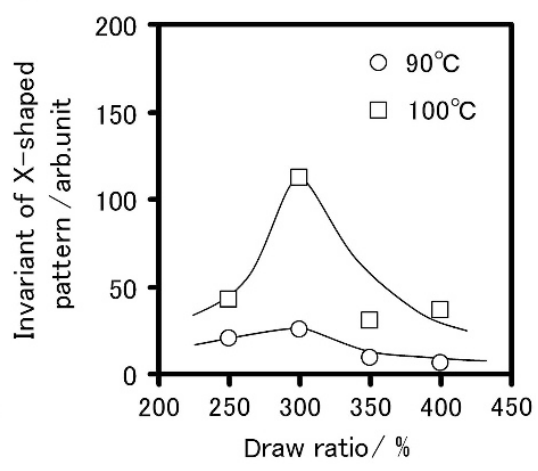

Figure 3 (a) Invariants of the 4-spot patterns and (b) the X-shaped patterns at various draw ratios.
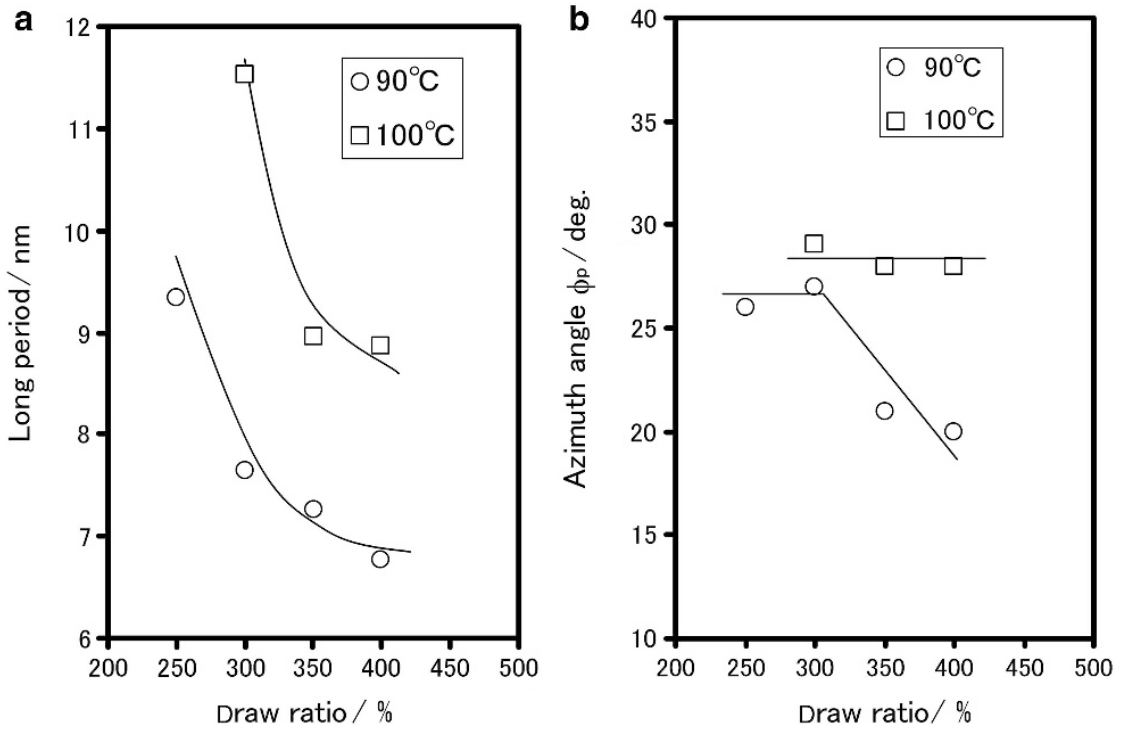

Figure 4 (a) The long period and (b) azimuthal angle $\phi_{\mathrm{p}}$ for the 4 -spot patterns at 90 and $100{ }^{\circ} \mathrm{C}$ as a function of the draw ratio.

it levels off above $300 \%$ at $90{ }^{\circ} \mathrm{C}$. Additionally, the invariant of the $\mathrm{X}$-shaped pattern has a maximum at $300 \%$ for 90 and $100{ }^{\circ} \mathrm{C}$, although the peak intensity is very weak, especially at $90^{\circ} \mathrm{C}$. This result implies that many lamellar crystals are formed in the range of draw ratios between 300 and $400 \%$ and that the newly formed lamellar crystals are inserted between the existing lamellar crystals, at least at $100^{\circ} \mathrm{C}$. In other words, the number of isolated single lamellar crystals decreases and hence the number of stacked lamellar crystals increases.

The long period $L$ was evaluated from Bragg's law, $L=2 \pi / q_{\max }$, where $q_{\max }$ is the peak position in the 4 -spot pattern and given by $q_{\max }=\left[q_{\mathrm{x}}^{2}+q_{\mathrm{y}}{ }^{2}\right]^{1 / 2}$. Figure $4 \mathrm{a}$ shows the long period of the 4 -spot pattern at 90 and $100^{\circ} \mathrm{C}$ as a function of the draw ratio. As was predicted from the insertion of the newly formed lamellar crystals, the long period $L$ decreases with the draw ratio. Similar results on the invariant and the long period for the 4-spot patterns were obtained from the in-situ measurements of PET during the uniaxial drawing process by Kawakami et al., ${ }^{5}$ suggesting that our samples are properly quenched to fix the PET structure in during the drawing. We have to mention the possibility of fragmentation of the lamellar crystals into small pieces during drawing, which may also make the long period smaller as the draw ratio increases.
Next, we have evaluated the azimuthal angle $\phi_{\mathrm{p}}$ of the peak position for the 4-spot pattern, which was defined as the angle between the peak position and the equator, namely, $\phi_{\mathrm{p}}=0$ and $90^{\circ}$ correspond to the lamellar surface normal to the draw direction and parallel to the draw direction, respectively. In other words, the azimuth angle $\phi_{\mathrm{p}}$ is the angle between the lamellar stacking direction and the direction perpendicular to the draw direction. The obtained azimuthal angle of the peak $\phi_{\mathrm{p}}$ in the 4-spot pattern was plotted in Figure $4 \mathrm{~b}$ as a function of the draw ratio. The azimuthal angle of the peak $\phi_{\mathrm{p}}$ decreases with the draw ratio from $\phi_{\mathrm{p}}=27^{\circ}$ to $20^{\circ}$ at $90^{\circ} \mathrm{C}$, while it remains nearly constant around $\phi_{\mathrm{p}}=28^{\circ}$ at $100^{\circ} \mathrm{C}$. The inclination angle of the surface of the lamellar crystal in PET is still an unsolved issue. According to the calculation of Asano et al., ${ }^{1}$ if the lamellar surface is fit to the shift of one monomer unit, the inclination angle should be $26^{\circ}$ in our definition of $\phi_{\mathrm{p}}$. This value is very close to the value of the azimuthal angles at the draw ratio of $300 \%$ at 90 and $100{ }^{\circ} \mathrm{C}$ (27 and $29^{\circ}$, respectively), implying that the 4-spot pattern of our samples must originate from the inclination of the lamellar surface due to the shift of one monomer unit. However, it is difficult to explain why the azimuthal angle $\phi_{\mathrm{p}}$ decreases with the draw ratio at $90^{\circ} \mathrm{C}$. Generally speaking, there are two possible explanations for that. One is the rotation of the whole stacked lamellar crystals, and 
the other is the decrease in the inclination angle $\phi_{\mathrm{p}}$ of the lamellar surface. At the moment, the latter is more plausible because it is difficult to imagine that the stacked direction rotates to the direction normal to drawing. However, we have no conclusions to make on the molecular picture from this observation.

\section{Simulation of 2D SAXS patterns}

Next, we investigated the details of the interesting but complicated shape of the 4-spot pattern. To investigate the shape, we tried to reproduce the observed SAXS patterns using a simple model that took



Figure 5 The model of the lamellar structure used in the calculation. into account the lamellar size, lamellar period, lamellar orientation and its distribution.

The lamellar model used in the calculation is illustrated in Figure 5. The draw direction is parallel to the vertical axis (y axis), and the stacking direction is defined by the angle $\phi$ in Figure 5. The intensity distribution for the lamellar structure $I\left(q_{\mathrm{x}}, q_{\mathrm{y}}, \phi=0\right)$ is calculated as follows:

$$
I\left(q_{x}, q_{y}, \phi=0\right) \propto \frac{\sin ^{2}\left(a q_{x} / 2\right)}{\left(a q_{x} / 2\right)^{2}} \cdot \frac{\sin ^{2}\left(b q_{y} / 2\right)}{\left(b q_{y} / 2\right)^{2}} \times\left|1+e^{i A q_{x}}\right|^{2}
$$

Here, $a$ and $b$ are the lamellar thickness parallel to the stacking direction of the lamellae and the lamellar length normal to the stacking direction of the lamellae, respectively, and $A$ is the long period. Note that the lamella length $b$ is distinguished from the lamella length $L_{\mathrm{x}}$. The former and the latter are the length in the model and the experimentally observed length, respectively. The first term on the right hand side of equation (1) is the form factor of the lamella, and the second term is the interference term between two lamellae. The intensity of each stacked lamellae with different stacking direction $\phi_{\mathrm{i}}$ was calculated and the total SAXS intensity $I\left(q_{\mathrm{x}}, q_{\mathrm{y}}\right)$ was obtained by summing up the contributions from all lamellae with weight fractions $w_{\mathrm{i}}$, which is given by

$$
I\left(q_{x}, q_{y}\right)=\sum_{i} w_{i} \cdot I_{i}\left(q_{x}, q_{y}, \phi_{i}\right)
$$

The comparison between the observed and calculated 2D SAXS patterns is shown in Figure 6 for the three 4-spot patterns for the
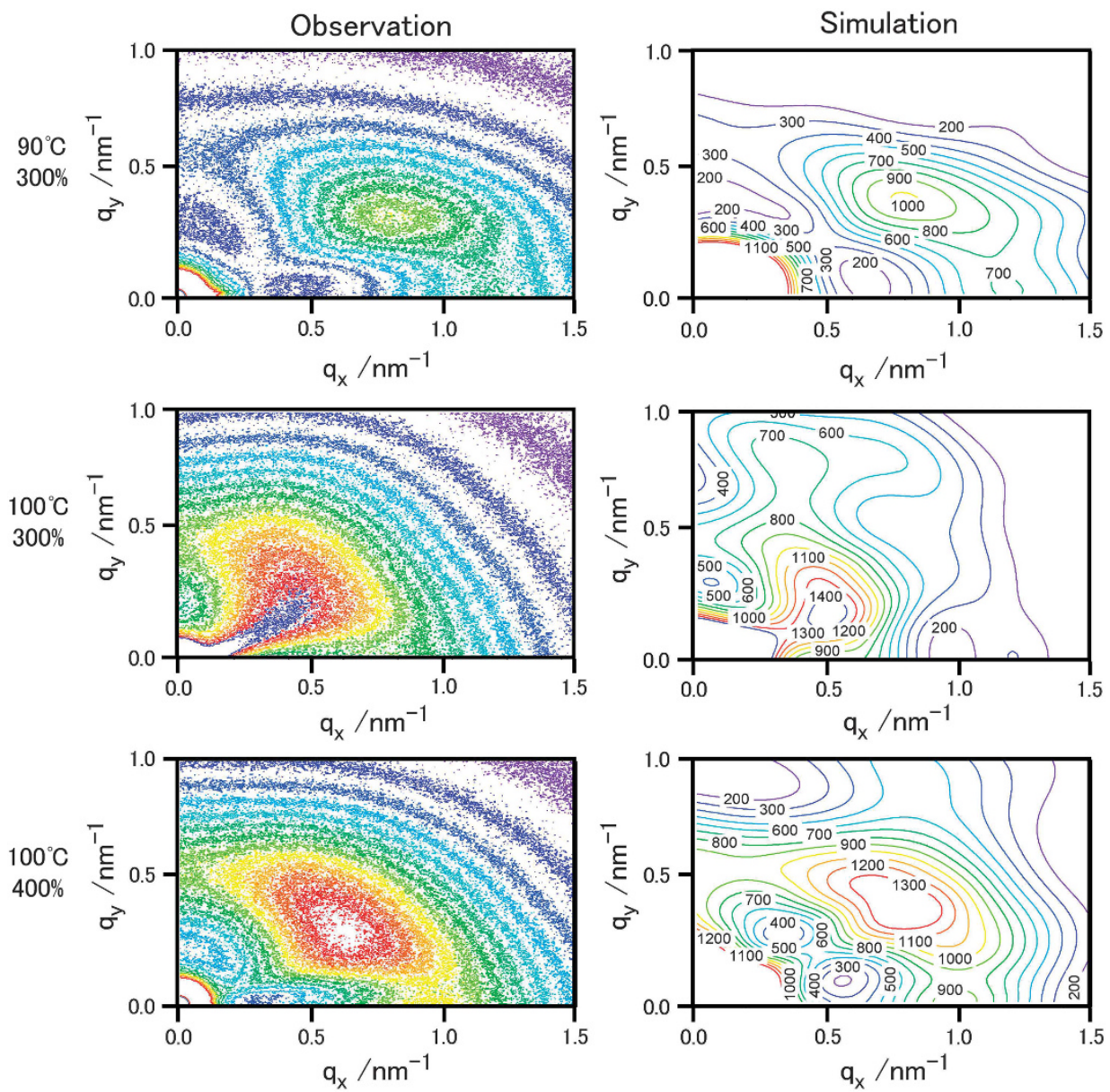

Figure 6 Comparison between the observed and calculated 4 -spot patterns for $300 \%$ at 90 and $100{ }^{\circ} \mathrm{C}$ and for $400 \%$ at $100{ }^{\circ} \mathrm{C}$. 
drawing ratios of $300 \%$ at 90 and $100{ }^{\circ} \mathrm{C}$ and of $400 \%$ at $100{ }^{\circ} \mathrm{C}$. The calculated patterns reproduced the complicated features of the observed 4-spot patterns rather well, except for near the origin in $q$-space. This is due to contributions from the form factors because we only considered the stacking of two lamellae.

We calculated the long period $L$ after averaging the contributions from all lamellae, the results are shown in Figure 7, to compare the observed and calculated long periods for the draw ratios of $300 \%$ at 90 and $100{ }^{\circ} \mathrm{C}$ and of $400 \%$ at $100{ }^{\circ} \mathrm{C}$. The temperature dependence and the draw ratio dependence are well reproduced in the calculation, suggesting that the calculation is correctly applied. However, the calculated long period is always shorter than the observed one. In this model calculation, we neglected the fluctuations in the mutual orientation of two stacked lamellae, and hence there is a valley between the first and second interference peaks. Therefore, to reproduce the observed 4 -spot pattern, the fractions of the stacked

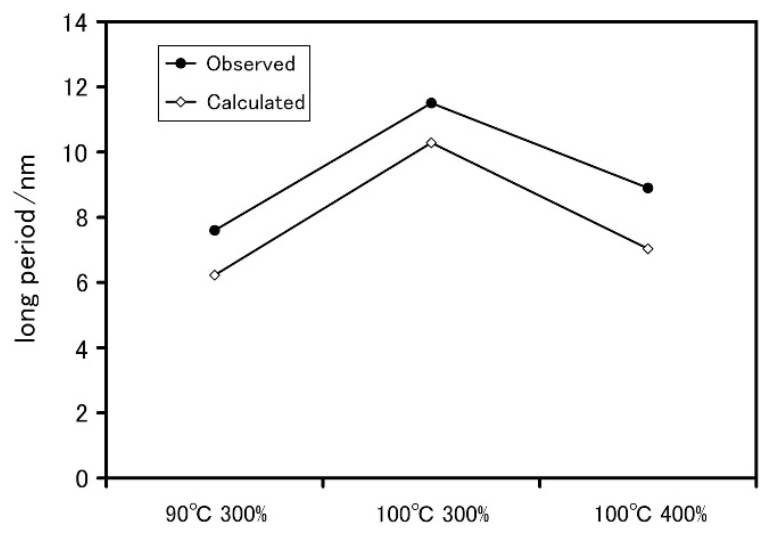

Figure 7 Comparison between the observed and calculated long periods. lamellae with the shorter long period $A$ are larger in the calculation than those in the observed data.

Distributions of the orientation angle and the size in the lamellae Based on the results of the calculation, we examined the orientation and the length of the lamellae in the 4 -spot pattern of PET. We calculated the area fraction of the lamellae in 2D space as a function of the stacking angle $\phi$. The area fraction $S_{i}$ of lamellae with a stacking angle $\phi_{\mathrm{i}}$ was calculated as follows.

$$
S_{i}=\frac{k \cdot w_{i} \cdot a_{i} \cdot b_{i}}{\sum_{i} k \cdot w_{i} \cdot a_{i} \cdot b_{i}}
$$

where $k=1$ and 2 for a single lamella and stacking lamellae, respectively. The calculated area fraction is shown in Figure 8a as a function of the stacking angle $\phi$ for $300 \%$ at 90 and $100{ }^{\circ} \mathrm{C}$ and for $400 \%$ at $100{ }^{\circ} \mathrm{C}$. Note that the contribution of the isolated single lamella is not included in Figure 8, meaning that the fraction in Figure 8 is that of the stacked lamellae in the total lamellae. First, we compare the data for $300 \%$ at $90{ }^{\circ} \mathrm{C}$ with that for $300 \%$ at $100{ }^{\circ} \mathrm{C}$. At $90^{\circ} \mathrm{C}$, the stacking direction is located at $\sim 25^{\circ}$, which is very close to the surface inclination angle $\left(\phi=26^{\circ}\right)$ predicted by Asano et al. ${ }^{1}$ suggesting that the 4 -spot pattern at $90^{\circ} \mathrm{C}$ originated from the inclination of the lamellar surface due to the shift of one monomer unit. Comparing the distribution of the stacking angle at $100^{\circ} \mathrm{C}$ for $300 \%$ with that at $90^{\circ} \mathrm{C}$, the distribution is very wide at $100^{\circ} \mathrm{C}$. This is the origin of the very large area in the 4 -spot pattern at $100^{\circ} \mathrm{C}$ (Figure 1). With an increase in the draw ratio from 300 to $400 \%$ at $100^{\circ} \mathrm{C}$, some lamellae appear in the low $\phi$ region below $\sim 20^{\circ}$, while the average peak position does not shift with the draw ratio (Figure 4b). Currently, it is unclear why lamellae appear in the low $\phi$ region as the draw ratio increases. However, it is clear that such a simple calculation provides information on the distribution of the stacking angles of the lamellae from the 2D 4-spot pattern.
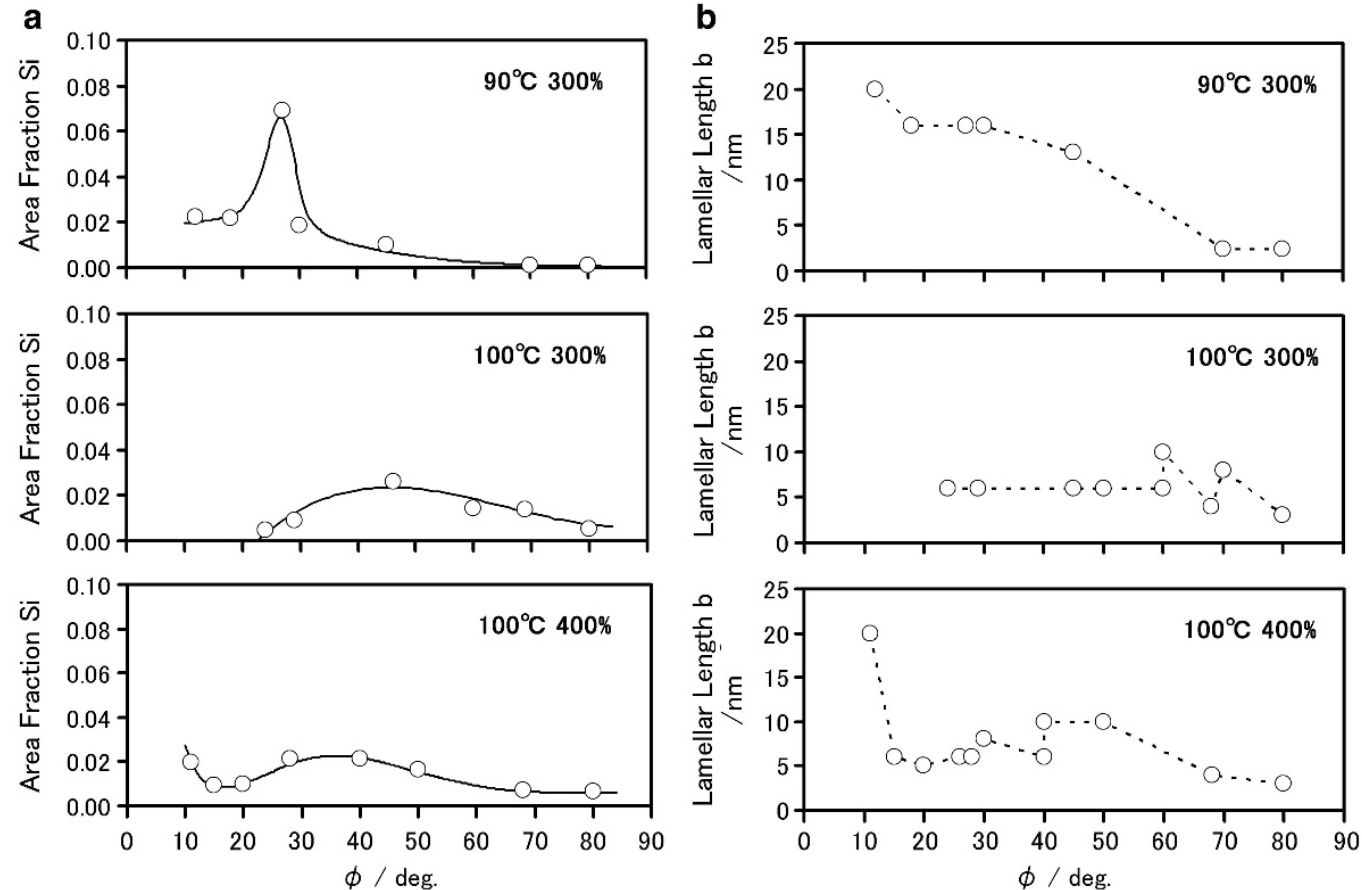

Figure 8 (a) The area fraction of the lamellae and (b) the lamellar length $b$ as a function of the stacking direction of the lamellae. 
In Figure $8 \mathrm{~b}$, the average lamellar length $b$ in the 4 -spot pattern is plotted against the stacking angle $\phi$. Comparing the lengths at 90 and $100{ }^{\circ} \mathrm{C}$ at the same draw ratio of $300 \%$, the lamellar length is much smaller in the drawn PET at $100^{\circ} \mathrm{C}$ than at $90^{\circ} \mathrm{C}$, and there are two potential physical reasons for this difference: one reason is that lamellae with larger lengths are formed at $90^{\circ} \mathrm{C}$ than at $100{ }^{\circ} \mathrm{C}$, and the other is that fragmentation of the lamellae into small pieces during the drawing process occurs more easily at $100{ }^{\circ} \mathrm{C}$ than at $90^{\circ} \mathrm{C}$. It is hard to refute either hypothesis at the moment. As mentioned above, the length of the isolated single lamella calculated from the $\mathrm{X}$-shaped pattern is $\sim 80 \mathrm{~nm}$, while the length evaluated from the 4-spot pattern, which corresponds to the stacked lamellae, is in the range of $5-20 \mathrm{~nm}$ (Figure $8 \mathrm{~b}$ ). The length of the isolated single lamellae is much larger than that of the stacked lamellae. This large length of the isolated single lamellar could be understood as follows. When a new isolated lamella is formed, it may be rather large because it is not fully crystallized but has a sort of intermediate structure similar to the smectic form, as shown by Asano et al., ${ }^{1}$ and it is fragmented when it is fully crystallized during the drawing process.

Regarding the dependence of the stacking angle $\phi$ on the length $b$, the length decreases with an increase in the stacking angle at $90^{\circ} \mathrm{C}$ This relationship suggests that the lamellar crystals stacked along the draw direction are more easily fragmented than those stacked parallel to the draw direction. As for the $100{ }^{\circ} \mathrm{C}$ data, the lamellar length is smaller than that at $90^{\circ} \mathrm{C}$ and almost independent of the stacking angle, suggesting that lamellar crystals with small lengths are formed during the drawing process, and the lamellae with small lengths are harder to fragment during further drawing. On the other hand, in the $400 \%$ data at $100^{\circ} \mathrm{C}$, lamellae with rather large lengths of $b \sim 20 \mathrm{~nm}$ are observed at $\phi \sim 10^{\circ}$, which was surprising to us. To explain the appearance of the large lamellae, we have to consider the formation of new lamellae. If this is true, the formation of new lamellae (or the nucleation and growth of lamellar crystals) occurs during the draw process from 300 to $400 \%$ at $100{ }^{\circ} \mathrm{C}$. Another possible explanation is that small lamellae disappear (or melt) and large ones grow (Ostwald ripening). The above discussions on the structural formation of PET still include some speculative parts; however, the present precise SAXS measurements and the simple calculation are useful for revealing many aspects of the crystallization process of PET during drawing.

\section{CONCLUSIONS}

We have studied the structural formation of PET during uniaxial drawing at temperatures of 90 and $100{ }^{\circ} \mathrm{C}$, which is above the glass transition temperature, using small-angle and wide-angle synchrotron radiation X-ray scattering (SAXS and WAXS) at the BL BL03XU at SPring-8. The high performance of the BL, such as the high intensity, wide-dynamic range and low background, enabled us to perform a very precise analysis of the $2 \mathrm{D}$ SAXS data. We could separately observe the X-shaped pattern and the so-called 4-spot pattern in the 2D SAXS pattern of the drawn PET, which are assigned to isolated single lamellae and stacked lamellae. Analyzing the data, we found that new lamellar crystals appeared during drawing and the isolated lamellae changed to stacked lamellae. This is one of the important findings in this paper. To extract information on the lamellae, we carried out a calculation of 2D SAXS patterns using a simple model that took into account the lamellar size, the lamellar spacing and the orientation distribution of the lamella stacking direction and succeeded in reproducing the observed complicated 2D SAXS patterns. Based on the results of the calculation, we discussed the distribution of the lamellar stacking direction to show that the distribution is very wide at $100{ }^{\circ} \mathrm{C}$ compared with that at $90^{\circ} \mathrm{C}$. We also discussed the lamellar length as a function of the stacking angle, reaching the conclusion that new lamellar crystals with rather large lengths ( $\sim 20 \mathrm{~nm}$ ) are formed during the drawing from 300 to $400 \%$. Thus, as was shown in the paper, the simple calculation and precise SAXS measurements were useful in studying the distributions of the orientation and the length in the stacked lamellae.

\section{ACKNOWLEDGEMENTS}

The authors thank Toray Industries and the Toray Research Center, Inc. in Japan for financial support. The authors are grateful to Dr Masunaga and Dr Ogawa for their kind support during the experiments at the FSBL BL03XU at SPring-8. The authors also thank Ms Watanabe for the sample preparation and the assistance in the SAXS image analysis.

1 Asano, T., Balta-Calleja, F. J., Flores, A., Tanigaki, M., Mina, M. F., Sawatari, C., Itagaki, H., Takahashi, H. \& Hatta, I. Crystalization of orientated amorphous poly(ethlene terephthalate) as revealed by X-ray diffraction and microhardness. Polymer 40, 6475-6484 (1999).

2 Kawakami, D., Ran, S., Burger, C., Fu, B., Sics, I. \& Hsiao, B. H. Mechanism of structural formation by uniaxial deformation in amorphous poly(ethylene terephthalate) above the glass temperature. Macromolecules 36, 9275-9280 (2003).

3 Kawakami, D., Ran, S., Burger, C., Fu, B., Sics, I., Chu, B. \& Kikutani, T. Structural formation of amorphous poly(ethylene terephthalate) during uniaxial deformation above glass temperature. Polymer 45, 905-918 (2004).

4 Kawakami, D., Hsiao, B. S., Burger, C., Ran, S., Avila-Orta, C., Sics, I., Kikutani, T. \& $\mathrm{Chu}, \mathrm{B}$. Deformation-induced phase transition and superstructure formation in poly(ethylene terephthalate). Macromolecules 38, 91-103 (2005).

5 Kawakami, D., Ran, S., Burger, C., Avila-Orta, C., Sics, I., Chu, B., Hsiao, B. H. \& Kikutani, T. Superstructure evolution in poly(ethylene terephthalate) during uniaxial deformation above glass transition temperature. Macromolecules 39, 2909-2920 (2006).

6 Kawakami, D., Burger, C., Ran, S., Avila-Orta, C., Sics, I., Chu, B., Chiao, C. M., Hsiao, B. H. \& Kikutani, T. New insights into lamellar structure development and SAXS/WAXS sequence appearance during uniaxial stretching of amorphous poly(ethylene terephthalate) above glass transition temperature. Macromolecules 41, 2859-2867 (2008).

7 Shimizu, J., Kikutani, T., Takaku, A. \& Okui, N. High speed melt spinning of poly(ethylene terephthalate). Sen'i Gakkaishi 37, T135-T142 (1981).

8 Shioya, M., Kawazoe, T., Okazaki, R., Suei, T., Sakurai, S., Yamamoto, K. \& Kikutani, T. Small-angle $x$-ray scattering study on the tensile fracture process of poly(ethylene terephthalate) fiber. Macromolecules 41, 4758-4765 (2008).

9 Mahendrasingam, A., Blundell, D. J., Martin, C., Fuller, W., MacKerron, D. H., Harvie, J. L., Oldman, R. J. \& Riekel, C. Influence of temperature and chain orientation on the crystallization of poly(ethylene terephthalate) during fast drawing. Polymer $\mathbf{4 1}$, 7803-7814 (2000)

10 Blundell, D. J., Mahendrasingam, A., Martin, C., Fuller, W., MacKerron, D. H., Harvie, J. L., Oldman, R. J. \& Riekel, C. Orientation prior to crystallisation during drawing of poly(ethylene terephthalate). Polymer 41, 7793-7802 (2000).

11 Mahendrasingam, A., Martin, C., Fuller, W., Blundell, D. J., Oldman, R. J., MacKerron, D. H., Harvie, J. L. \& Riekel, C. Observation of a transient structure prior to straininduced crystallization in poly(ethylene terephthalate). Polymer 41, 1217-1221 (2000).

12 Mahendrasingam, A., Blundell, D. J., Wright, A. K., Urban, V., Narayaman, T. \& Fuller W. Observations of structure development during crystallisation of oriented poly(ethylene terephthalate). Polymer 44, 5915-5925 (2003).

13 Yamaguchi, T., Mutara, T., Koide, M., Hitoosa, S., Urakawa, H., Ohkoshi, Y., Gotoh, Y. Magura, M., Kotera, M. \& Kajiwara, K. Initial stage of fiber structure development in the continuous drawing of poly(ethylene terephthalate). J. Polym. Sci. B Polym. Phys. 46, 2126-2142 (2008)

14 Rule, R. J., MacKerron, D. H., Mahendrasingam, A., Martin, C. \& Nyet, T. M. W. Timeresolved two-dimensional small angle $\mathrm{x}$-ray scattering studies of oriented poly(ethylene terephthalate) (PET) using paracrystalline modeling techniques. Macromolecules 28, 8517-8522 (1995)

15 Daubeny, R., de, P., Bunn, C. W. \& Brown, C. J. The crystal structure of polyethylene terephthalate. Proc. Roy. Soc. Ser A 226, 531-542 (1954).

16 Masunaga, H., Ogawa, H., Takano, T., Sasaki, S., Goto, S., Tanaka, T., Seike, T. Takahashi, S., Takeshita, K., Nariyama, N., Ohashi, H., Ohata, T., Furukawa, Y. Matsushita, T., Ishizawa, Y., Yagi, N., Takata, M., Kitamura, H., Sakurai, K., Tashiro, K., Takahara, A., Amamiya, A., Horie, K., Takenaka, M., Kanaya, T., Jinnai, H., Okuda, H., Akiba, I., Takahashi, I., Yamamoto, K., Hikosaka, M., Sakurai, H., Shinohara, Y. Okada, A. \& Sugihara, Y. Multipurpose soft-material SAXS/WAXS/GISAXS beamline at SPring-8. Polym. J. 43, 471-477 (2011).

17 Mandelkern, L. Crystallization of Polymers: Kinetics and Mechanisms. Vol 2. (Cambridge University Press, Cambridge, UK, 2004).

18 Guinier, A. \& Fournet, G. Small-Angle Scattering of X-Rays. (John Wiley \& Sons, Inc. New York, 1955). 